\title{
Current development of chimeric antigen receptor T-cell therapy
}

\author{
Jiasheng Wang ${ }^{1}$, Yongxian $\mathrm{Hu}^{2,3,4}$, He Huang ${ }^{2,3,4}$ \\ ${ }^{1}$ Department of Internal Medicine, MetroHealth Medical Center, Case Western Reserve University, Cleveland, OH, USA; ${ }^{2}$ Bone Marrow \\ Transplantation Center, the First Affiliated Hospital, School of Medicine, Zhejiang University, Hangzhou 310058, China; ${ }^{3}$ Institute of Hematology, \\ Zhejiang University, Hangzhou 310058, China; ${ }^{4}$ Zhejiang Province Engineering Laboratory for Stem Cell and Immunity Therapy, Hangzhou \\ 310058, China \\ Contributions: (I) Conception and design: All authors; (II) Administrative support: None; (III) Provision of study material or patients: None; (IV) \\ Collection and assembly of data: All authors; (V) Data analysis and interpretation: All authors; (VI) Manuscript writing: All authors; (VII) Final \\ approval of manuscript: All authors. \\ Correspondence to: He Huang, MD, PhD. Bone Marrow Transplantation Center, The First Affiliated Hospital, School of Medicine, Zhejiang \\ University, No.79 Qingchun Road, Hangzhou 310058, China. Email: huanghe@zju.edu.cn.
}

\begin{abstract}
Chimeric antigen receptor (CAR) T-cell therapy has achieved great success in recent years, with encouraging complete remission rate and long-term durability of response, especially in advanced B-cell malignancies. With the approval of tisagenlecleucel and axi-cel by FDA to treat refractory/relapsed acute lymphoblastic leukemia and non-Hodgkin lymphoma, our understanding of CAR T cells has been progressing rapidly. In this review, we discussed the designs of CAR T cells, factors affecting response, adverse effects, as well as application beyond B-cell malignancies.
\end{abstract}

Keywords: Chimeric antigen receptor (CAR); cytokine release syndrome (CRS); neurologic toxicity; acute lymphoblastic leukemia (ALL); non-Hodgkin lymphoma (NHL)

Received: 15 July 2018; Accepted: 11 November 2018; Published: 03 December 2018.

doi: $10.21037 /$ sci.2018.11.05

View this article at: http://dx.doi.org/10.21037/sci.2018.11.05

\section{Introduction}

Chimeric antigen receptor (CAR) T-cell therapy has revolutionized the treatment of relapsed/refractory $(\mathrm{r} / \mathrm{r})$ B-cell malignancies. We have seen encouraging complete remission (CR) rate and long-term durability of response in $\mathrm{r} / \mathrm{r}$ acute lymphoblastic leukemia (ALL) and nonHodgkin lymphoma (NHL). Acting as a "living drug", CAR $\mathrm{T}$ cells are engineered $\mathrm{T}$ cells from patients or previous donors, which can recognize tumor antigens in a major histocompatibility complex (MHC) independent manner.

With the invention of retroviral vectors, $\mathrm{T}$ cell engineering began around 1990. The first generation of CAR T cells were developed in 1993 (1) (Figure 1), however, they are not clinically effective due to short persistence. In 1998, the introduction of co-stimulatory domain (2) paved the way for today's success. In 2003, second-generation CARs (Figure 1) were built to target CD19, which set the stage for the first successful treatment of a patient with ALL in 2011.

With the FDA approval of tisagenlecleucel in August 2017 for children and young adults with r/r ALL, and axi-cel in October 2017 for adults with $\mathrm{r} / \mathrm{r}$ NHL, the research of CAR T-cell therapy has entered a new era. Various new designs and strategies are under development to further boost the efficacy and control the adverse effects of CAR T cells. This mini-review will focus on the current understanding of CAR T cells, discussing the designs, durability of response, adverse effects, as well as its application in other malignant diseases.

\section{Designs of CAR T cells}

CARs consist of an ectodomain of single chain variable fragment $(\mathrm{scFv})$ to recognize tumor antigen, an endodomain with signaling modules and domains from $\mathrm{CD} 3 \zeta$, and a spacer and a transmembrane domain connecting the extracellular and intracellular parts. The affinity of $\mathrm{scFv}$ 


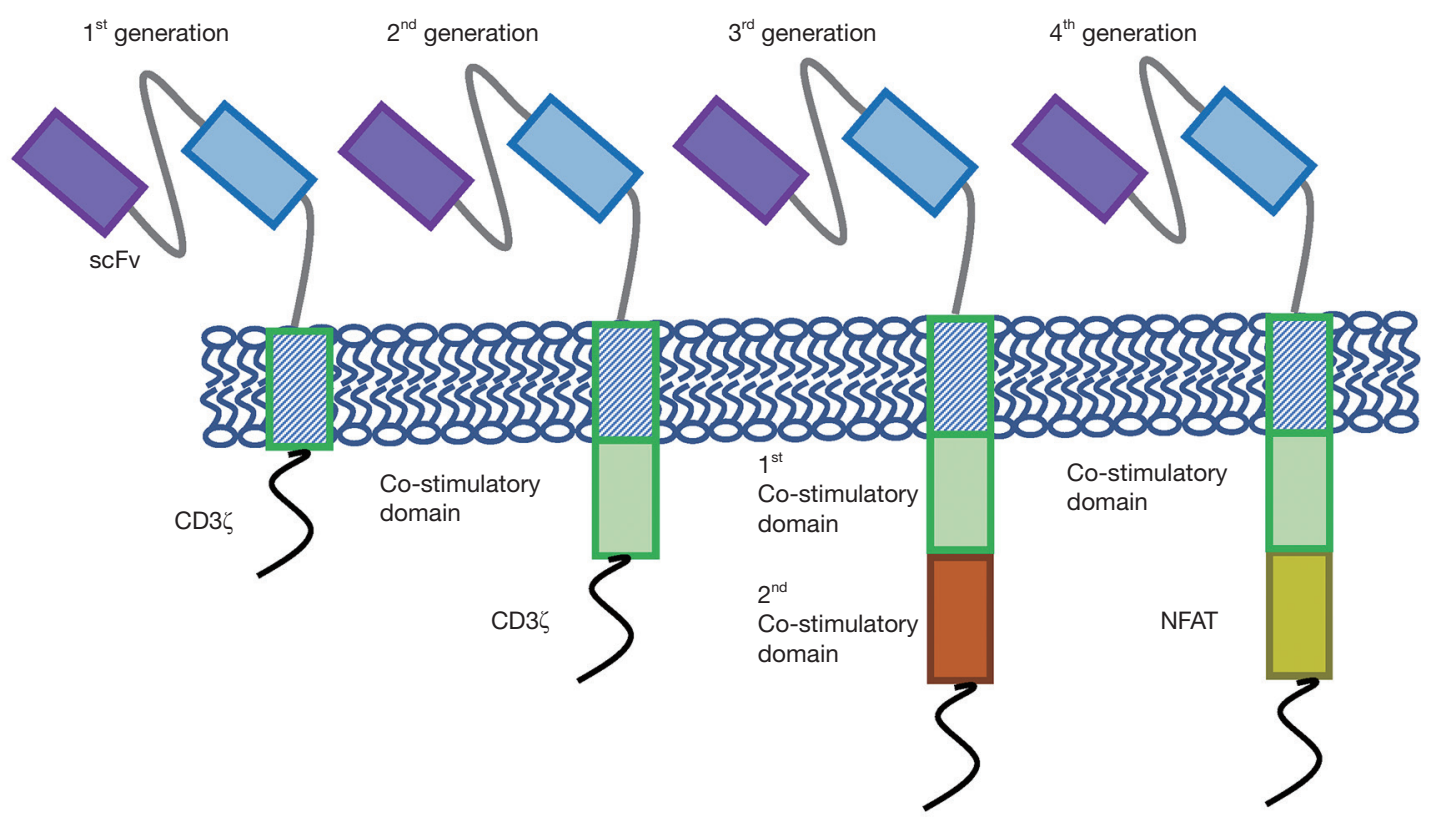

Figure 1 Generations of CAR T cells. All generations of CARs have an ectodomain of single chain variable fragment (scFv), an endodomain with signaling modules and domains from $\mathrm{CD} 3 \zeta$, and a transmembrane domain connecting the extracellular and intracellular parts. Different generations differ on the endodomain. For the $1^{\text {st }}$ generation, the endodomain is the CD $3 \zeta$. The $2^{\text {nd }}$ generation has co-stimulatory domain and $\mathrm{CD} 3 \zeta$ combined, which improved CAR T cell in vivo persistence. The $3^{\text {rd }}$ generation has two consecutive co-stimulatory domains. For the 4th generation, the co-stimulatory domain was combined with the nuclear factor of activated T-cells (NFAT) domain, which are transcription factors that can control cytokine production. CAR, chimeric antigen receptor; DC, dendritic cell.

influences CAR T-cell function (3); moreover, it could be designed to recognize two or more antigens (tandem CAR or bispecific CAR), rendering CAR $\mathrm{T}$ cells to be activated by either or both antigens (4-6). The transmembrane domain which is usually derived from $\mathrm{CD} 3 \zeta$, $\mathrm{CD} 4, \mathrm{CD} 8$, or CD28 molecules, also plays an indispensable role in signal transduction (7). The development of CAR T cells have gone through certain generations (Figure 1), which differs mainly in the endodomain. The first generation of CAR T cells only harbors $\mathrm{CD} 3 \zeta$ domain, which lacks persistence. The incorporation of co-stimulatory molecules CD28 and 4-1BB into the intracellular signaling domains significantly promoted CAR T-cell expansion and persistence, leading to today's breakthrough. CARs harboring two consecutive co-stimulatory domains are called the third-generation CARs. Furthermore, CARs have also been combined with other accessory proteins, such as chemokines (8) and inducible killing switch (9), to improve the function and safety, leading to the so-called fourth generation. Despite the different designs, final activation is initiated by Lckmediated phosphorylation of CD3 immunoreceptor tyrosine-based activation motifs (10).
The CAR transgene is usually introduced into the genome randomly via $\gamma$ retroviral transduction, lentiviral transduction, or sleeping beautify system (11). However, random insertion may lead to disruptions of important genes. For example, Fraietta et al. described a case where CAR insertion disrupted TET2 gene (12). Although the accident promoted CAR T-cell proliferation and led to remission, it also carried the risk of oncogenesis. Moreover, the expression of randomly inserted $C A R$ is poorly regulated. Therefore, targeted insertion is preferred. For example, Eyquem et al. used CRISPR/Cas9 system to direct $C A R$ gene to the T-cell receptor $\alpha$ constant (TRAC) locus, which vastly enhanced CAR T-cell potency (13).

\section{Durable response}

CAR T-cell therapy was able to achieve high CR rate in heavily pretreated patients with refractory or relapsed diseases. For ALL, Park et al. showed that in 53 patients a CR rate of $83 \%$ could be reached (14). For diffuse large B-cell lymphoma (DLBCL), the most recent data from the ZUMA-1 study at the 2018 American Society of Clinical 
Oncology (ASCO) annual meeting showed that the overall response rate (ORR) at 15.1 months was $79 \%$, with CR rate of $58 \%(15)$.

In addition to high CR rate, CAR T-cell therapy has demonstrated durable response, as evidenced by improved survival compared with traditional chemotherapy. For ALL, Park et al. showed that after a median follow-up of 29 months, the median event-free survival (EFS) was 6.1 months, the median overall survival (OS) was 12.9 months (14). For DLBCL, Locke et al. showed that the OS at 18 months was $52 \%$ (ZUMA-1) (15). It is becoming clear that patients who maintained CR at month 3 or 6 are likely to experience long-term remission. Some studies have tried to identify factor that could predict the durability of response. For ALL, low disease burden and achievement of CR without minimal residual disease (MRD) after CAR T-cell therapy, but not better CAR T-cell expansion and long-term persistence was associated with better long-term outcome (16). Moreover, Hay et al. reported that patients with normal LDH level prior to lymphodepletion, a platelet count of at least $100 \mathrm{U} / \mathrm{L}$ prior to lymphodepletion, and receipt of cyclophosphatidic/fludarabine lymphodepletion regimen were independent predictors of better disease-free survival (DFS) (17). For DLBCL, as reported at the 2018 ASCO meeting, low International Prognostic Index (IPI) score, indolent histology, low lymph node burden, better CAR T-cell expansion and persistence, and higher CD8+ CAR T-cell counts were associated with better outcomes (18). Further studies are needed to confirm these factors in order to better stratify the risks of patients.

One important factor impacting long-term outcome is relapse after CAR T-cell therapy (19). During the 2018 ASCO meeting, Pillai et al. reported that in 150 ALL patients treated with CAR T-cells, 20 had CD19-positive relapses and 33 had CD19 negative relapses (20). Various mechanisms such as CD19 genetic and transcriptional alterations (21-23), lineage switch to myeloid leukemia (22), limited CAR T-cell expansion and persistence (24), and upregulation of immunosuppressive molecules such as PD-1/IDO $(11,25)$ have been identified to cause relapses. Strategies like com bination with PD-1 blockade (26), knockout of CAR T-cell PD-1 gene $(27,28)$, targeting multiple surface markers $(5,6)$ have been implicated.

\section{Adverse effects of CAR T-cell therapy}

The two most commonly observed acute adverse effects of CAR T-cell therapy are cytokine release syndrome
(CRS) and neurologic toxicity. CRS, the most commonly encountered adverse effect (29), presented as high fever, hypotension, and multi-organ toxicity. It is triggered by activation of CAR T cells and bystander immune cells, upon CAR engagement with antigens expressed by tumor cells. It is characterized by increased levels of various cytokines, including CAR T-cell-derived INF- $\gamma$ and IL-2, and monocyte-derived IL-6 and IL-1 (30,31) (Figure 2A). Different grading system has been proposed to describe the severity of CRS. The two most commonly used are the UPenn grading scale (32) and the scale proposed by Lee et al. (33) (Table 1). For grade 1 CRS, supportive care alone is usually adequate. For patients with higher grade CRS, more aggressive intervention with combination of anti-IL-6 therapy and/or steroids can usually result in satisfactory control. Recent studies (30,31) using murine models suggested that macrophage played a pivotal role in the CRS; administration of antagonists of macrophagederived cytokines such as IL-1 and IL-6, or diminishing macrophage function by blocking iNOS, could abate or even prevent the occurrence of CRS.

Neurologic toxicity, typically presented as confusion, delirium, seizure and cerebral edema, is the second most common acute adverse effect. It can occur concurrently with CRS or have a delayed onset even weeks after infusion. The mechanism of neurologic toxicity still remains unclear; it could be caused by passive diffusion of cytokines into the brain (35) or trafficking of CAR T cells into the central nervous system (CNS) (36). A recent study utilized a rhesus macaque model showed that neurologic toxicity was associated with increased levels of pro-inflammatory cytokines and pan- $\mathrm{T}$ cell infiltration (both CAR T cells and non-CAR T cells) (37) (Figure 2B). Pathology of patients with severe neurologic toxicity revealed endothelial activation, with capillary leak and increased blood-brain barrier (BBB) permeability (38). Neurologic toxicity can be graded based on the Common Terminology Criteria for Adverse Events (CTCAE) criteria or the grading system proposed by Neelapu et al. (29) (Table 1). For the management of neurologic toxicity, anti-IL-6 tocilizumab is believed ineffective without concurrent CRS, because this antibody is unable to cross BBB. The effectiveness of IL-1 receptor blocker in neurologic toxicity is still under examination (31).

CAR T-cell therapy can also cause other adverse effects, such as cytopenia, on-target/off-tumor recognition, and graft-versus-host disease if CAR T cells are allogeneic (39). At last, it is worth noting that toxicity varies in different 
Table 1 Common grading systems for cytokine release syndrome and neurologic toxicity

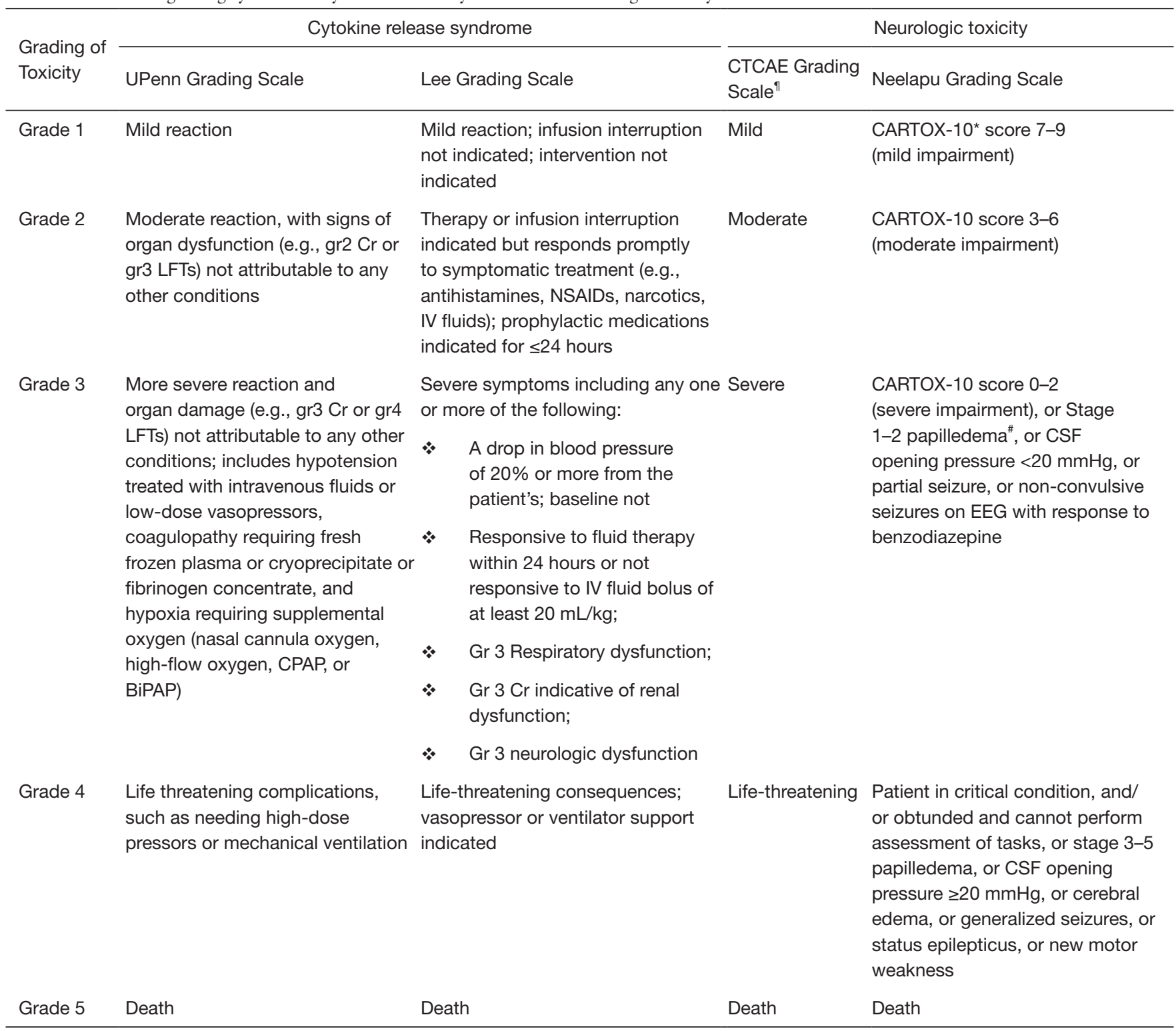

\footnotetext{
", in terms of levels of consciousness, orientation, ability to perform activities of daily living (in the con-text of encephalopathy), speech, tremors, seizures, incontinence, and motor weakness. The final grade is the highest grades of all the items evaluated; *, CARTOX-10 scale: one point is assigned for each of the following tasks that is performed correctly (normal cognitive function is defined by an overall score of 10): orientation to year, month, city, hospital, and President/Prime Minister of country of residence (total of 5 points); name three objects for example, point to clock, pen, button (maximum of 3 points); write a standard sentence, for example, 'our national bird is the bald eagle' (1 point); count backwards from 100 in tens (1 point); \#, papilledema grading is performed according to the modified Frisén scale (34). CTCAE, Common Terminology Criteria for Adverse Events; Gr, grade; Cr, creatinine; LFT, liver function test; CARTOX-10, CAR-T-cell-therapy-associated toxicity 10-point neurological assessment. CSF, cerebrospinal fluid.
} 

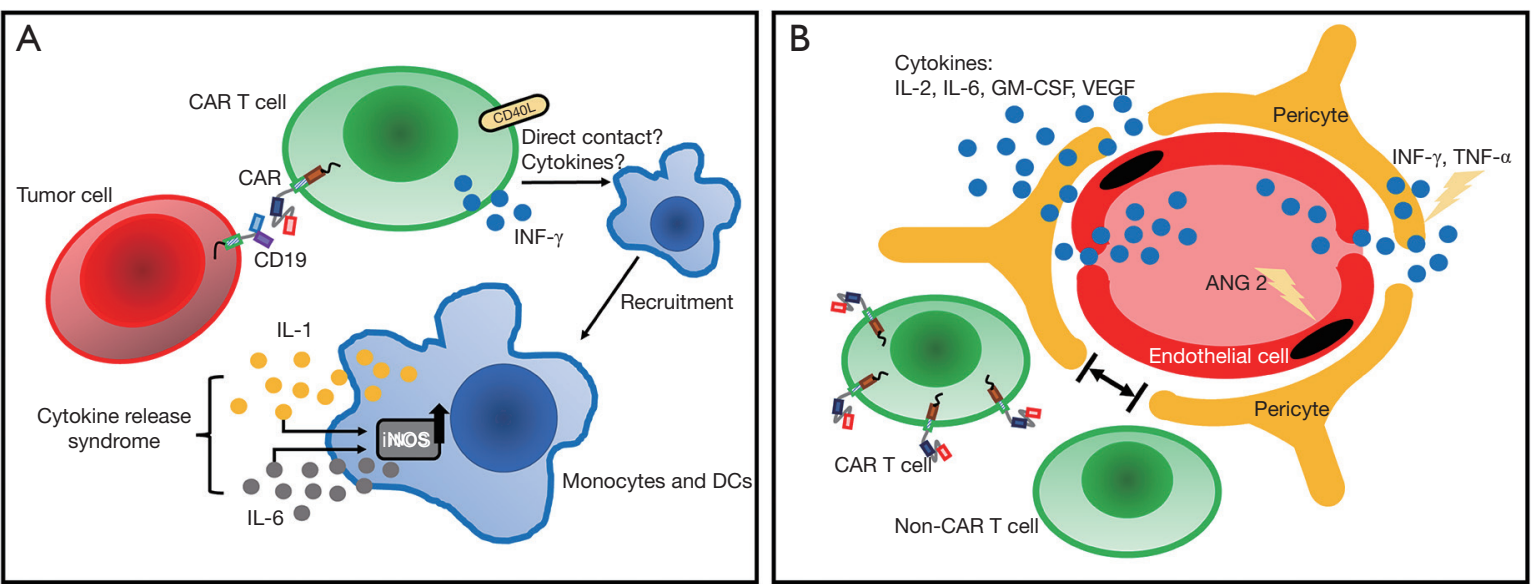

Figure 2 Models of CRS and neurologic toxicities. (A) CRS is mediated by CAR T cells and bystander immune cells such as monocytes and DCs. In the peripheral blood, when CAR T cells and tumor cells are engaged, CAR T cells are activated and start recruiting monocytes through direct contact such as CD40L-CD40 interaction, or through T-cell derived cytokines such as INF- $\gamma$. Activated monocytes can secrete key CRS cytokines IL-1 and IL-6, which in addition to cause symptoms of CRS, can further activate monocytes through iNOS. (B) Neurologic toxicities are caused by cytokines and/or CAR T and non-CAR T cells, secondary to increased BBB permeability. BBB is comprised of ECs and pericytes. Following increased levels of cytokines during CRS, ECs are activated with secretion of angiopoietin 2 (ANG2), which further promotes endothelial activation and microvascular permeability. Next, cytokines such as INF- $\gamma$ and TNF- $\alpha$ can cross ECs and cause pericyte stress, further increasing BBB permeability. Analysis of CSF showed high levels of IL-2, IL-6, GM-CSF and VEGF, as well as CAR T cells and non-CAR T cells. CRS, cytokine release syndrome; CAR, chimeric antigen receptor; DC, dendritic cell; iNOS, inducible nitric oxide synthase; BBB, blood-brain barrier; EC, endothelial cells; CSF, cerebrospinal fluid.

CAR T-cell designs and in different disease types (40).

\section{CAR T-cell therapy beyond B-cell malignancies}

Although CAR T-cell therapy is currently approved to treat only relapsed/refractory ALL and NHL, evidence of its effectiveness in other malignancies is emerging. For example, B-cell mature antigen (BCMA)-targeting CAR $\mathrm{T}$ cells have shown promising results in patients with multiple myeloma (MM). At the 2017 ASCO meeting, a single armed clinical trial reported 18 (95\%) of 19 patients achieved CR or near CR throughout a median follow-up of 208 days (41). In another report, Brudno et al. (42) showed an ORR of $81 \%$, with $63 \%$ CR or near CR. Despite the promising results, one of the biggest concerns of BCMAtargeting CAR $\mathrm{T}$ cells is that BCMA can be cleaved from cell surface and shed into blood, resulting in antigen loss. Indeed, BCMA loss has been reported in some studies $(42,43)$.

For acute myeloid leukemia, researchers are yet able to replicate the success in ALL and MM. The biggest challenge we are facing is to identify the optimal myeloid antigen, without causing significant on target/off tumor toxicity. Various targets, such as CD33, CD123, NKG2DL were currently examined in phase I clinical trials (44).

The success of CAR T cells in hematologic malignancies has yet to be extrapolated to solid tumors due to the lack of specific targetable antigens, on-target/off-tumor effect and the complex tumor microenvironment (45). Indeed, significant solid tumor regression without severe toxicities has yet to be reported except in one case where glioblastoma was successfully treated with intracranial IL13R $\alpha 2$-targeting CAR T cells (46). Different strategies, such as regional delivery of CAR T cells $(47,48)$, design of combinationaltargeting CARs (49), engineering CAR T cells to directly deliver cytokines within the tumor (50), combination with checkpoint inhibitors (51), and combination with oncolytic virus therapy $(52,53)$, have shown promising results in pre-clinical studies.

\section{Future perspectives}

With the approval of CAR T-cell therapy in advanced $\mathrm{B}$-cell malignancies, our understanding of CAR T-cell 
therapy has been progressing rapidly. With longer followups and more patients receiving CAR T-cell therapy, we would be able to stratify the risks in patients and predict the clinical outcomes. New therapeutic targets in the CRS and neurologic toxicities, such as IL-1 and iNOS, have been identified in pre-clinical studies; their roles should be confirmed in future research. Anyhow, we are walking toward more efficient and safer CAR T cells. At last, the success of CAR T-cell therapy in hematological malignancies has yet to be replicated in solid tumors. It requires great effort to tackle the unique challenges in solid tumors.

\section{Acknowledgements}

Funding: This work was supported by the grants from 973 Program (grant No. 2015CB964900), the Natural Science Foundation of China (grant No. 81230014, 81470341, $81520108002,81500157)$, Key Project of Science and Technology Department of Zhejiang Province (grant No. 2015C03G2010091).

\section{Footnote}

Conflicts of Interest: The authors have no conflicts of interest to declare.

\section{References}

1. Eshhar Z, Waks T, Gross G, et al. Specific activation and targeting of cytotoxic lymphocytes through chimeric single chains consisting of antibody-binding domains and the gamma or zeta subunits of the immunoglobulin and T-cell receptors. Proc Natl Acad Sci 1993;90:720-4.

2. Krause A, Guo HF, Latouche JB, et al. Antigendependent CD28 signaling selectively enhances survival and proliferation in genetically modified activated human primary T lymphocytes. J Exp Med 1998;188:619-26.

3. Hudecek M, Lupo-Stanghellini MT, Kosasih PL, et al. Receptor affinity and extracellular domain modifications affect tumor recognition by ROR1-specific chimeric antigen receptor T cells. Clin Cancer Res 2013;19:3153-64.

4. Grada Z, Hegde M, Byrd T, et al. TanCAR: A Novel Bispecific Chimeric Antigen Receptor for Cancer Immunotherapy. Mol Ther Nucleic Acids 2013;2:e105.

5. Hegde $M$, Mukherjee $M$, Grada Z, et al. Tandem CAR T cells targeting HER2 and IL13R $\alpha 2$ mitigate tumor antigen escape. J Clin Invest 2016;126:3036-52.
6. Zah E, Lin MY, Silva-Benedict A, et al. T Cells Expressing CD19/CD20 Bispecific Chimeric Antigen Receptors Prevent Antigen Escape by Malignant B Cells. Cancer Immunol Res 2016;4:498-508.

7. Bridgeman JS, Ladell K, Sheard VE, et al. CD3 $\zeta$-based chimeric antigen receptors mediate $\mathrm{T}$ cell activation via cis- and trans-signalling mechanisms: implications for optimization of receptor structure for adoptive cell therapy. Clin Exp Immunol 2014;175:258-67.

8. Harris DT, Kranz DM. Adoptive T Cell Therapies: A Comparison of $\mathrm{T}$ Cell Receptors and Chimeric Antigen Receptors. Trends Pharmacol Sci 2016;37:220-30.

9. Pegram HJ, Lee JC, Hayman EG, et al. Tumor-targeted T cells modified to secrete IL-12 eradicate systemic tumors without need for prior conditioning. Blood 2012;119:4133-41.

10. Di Stasi A, Tey SK, Dotti G, et al. Inducible apoptosis as a safety switch for adoptive cell therapy. $\mathrm{N}$ Engl J Med 2011;365:1673-83.

11. Kebriaei P, Singh H, Huls MH, et al. Phase I trials using Sleeping Beauty to generate CD19-specific CAR T cells. J Clin Invest 2016;126:3363-76.

12. Fraietta JA, Nobles CL, Sammons MA, et al. Disruption of TET2 promotes the therapeutic efficacy of CD19-targeted T cells. Nature 2018;558:307-12.

13. Eyquem J, Mansilla-Soto J, Giavridis T, et al. Targeting a CAR to the TRAC locus with CRISPR/Cas9 enhances tumour rejection. Nature 2017;543:113-7.

14. Park JH, Rivière I, Gonen M, et al. Long-Term Followup of CD19 CAR Therapy in Acute Lymphoblastic Leukemia. N Engl J Med 2018;378:449-59.

15. Locke FL, Ghobadi A, Jacobson CA, et al. Durability of response in ZUMA-1, the pivotal phase 2 study of axicabtagene ciloleucel (Axi-Cel) in patients (Pts) with refractory large B-cell lymphoma. J Clin Oncol 2018;36:abstr 3003.

16. Locke FL, Neelapu SS, Bartlett NL, et al. Phase 1 Results of ZUMA-1: A Multicenter Study of KTE-C19 AntiCD19 CAR T Cell Therapy in Refractory Aggressive Lymphoma. Mol Ther 2017;25:285-95.

17. Hay KA, Gauthier J, Hirayama AV, et al. Factors impacting disease-free survival in adult B cell B-ALL patients achieving MRD-negative CR after CD19 CAR-T cells. J Clin Oncol 2018;36:abstr 7005.

18. Gauthier J, Hirayama AV, Hay KA, et al. Factors associated with duration of response after CD19-specific CAR-T cell therapy for refractory/relapsed B-cell non-Hodgkin lymphoma. J Clin Oncol 2018;36:abstr 7567. 
19. Wang J, Hu Y, Huang H. Acute lymphoblastic leukemia relapse after CD19-targeted chimeric antigen receptor T cell therapy. J Leukoc Biol 2017;102:1347-56.

20. Pillai V, Rosenthal J, Muralidharan K, et al. Correlation of pre-CAR CD19 expression with responses and relapses after CAR T cell therapy. J Clin Oncol 2018;36:abstr 3051.

21. Sotillo E, Barrett DM, Black KL, et al. Convergence of Acquired Mutations and Alternative Splicing of CD19 Enables Resistance to CART-19 Immunotherapy. Cancer Discov 2015;5:1282-95.

22. Jacoby E, Nguyen SM, Fountaine TJ, et al. CD19 CAR immune pressure induces B-precursor acute lymphoblastic leukaemia lineage switch exposing inherent leukaemic plasticity. Nat Commun 2016;7:12320.

23. Fischer J, Paret C, El Malki K, et al. CD19 Isoforms Enabling Resistance to CART-19 Immunotherapy Are Expressed in B-ALL Patients at Initial Diagnosis. J Immunother 2017;40:187-95.

24. Turtle CJ, Hanafi LA, Berger C, et al. CD19 CAR-T cells of defined CD4+:CD8+ composition in adult B cell ALL patients. J Clin Invest 2016;126:2123-38.

25. Ninomiya S, Narala N, Huye L, et al. Tumor indoleamine 2,3-dioxygenase (IDO) inhibits CD19-CAR T cells and is downregulated by lymphodepleting drugs. Blood 2015;125:3905-16.

26. Chong EA, Melenhorst JJ, Lacey SF, et al. PD-1 blockade modulates chimeric antigen receptor (CAR)-modified $\mathrm{T}$ cells: refueling the CAR. Blood 2017;129:1039-41.

27. Ren J, Liu X, Fang C, et al. Multiplex Genome Editing to Generate Universal CAR T Cells Resistant to PD1 Inhibition. Clin Cancer Res 2017;23:2255-66.

28. Rupp LJ, Schumann K, Roybal KT, et al. CRISPR/Cas9mediated PD-1 disruption enhances anti-tumor efficacy of human chimeric antigen receptor T cells. Sci Rep 2017;7:737.

29. Neelapu SS, Tummala S, Kebriaei P, et al. Chimeric antigen receptor T-cell therapy - assessment and management of toxicities. Nat Rev Clin Oncol 2018;15:47-62.

30. Giavridis T, van der Stegen SJC, Eyquem J, et al. CAR $\mathrm{T}$ cell-induced cytokine release syndrome is mediated by macrophages and abated by IL-1 blockade. Nat Med 2018;24:731-8.

31. Norelli M, Camisa B, Barbiera G, et al. Monocyte-derived IL-1 and IL-6 are differentially required for cytokinerelease syndrome and neurotoxicity due to CAR T cells. Nat Med 2018;24:739-48.

32. Porter DL, Hwang WT, Frey NV, et al. Chimeric antigen receptor $\mathrm{T}$ cells persist and induce sustained remissions in relapsed refractory chronic lymphocytic leukemia. Sci Transl Med 2015;7:303ra139.

33. Lee DW, Gardner R, Porter DL, et al. Current concepts in the diagnosis and management of cytokine release syndrome. Blood 2014;124:188-95. Erratum in: Blood 2015;126:1048. Dosage error in article text.

34. Frisén L. Swelling of the optic nerve head: a staging scheme. J Neurol Neurosurg Psychiatry 1982;45:13-8.

35. Lee DW, Kochenderfer JN, Stetler-Stevenson M, et al. T cells expressing CD19 chimeric antigen receptors for acute lymphoblastic leukaemia in children and young adults: a phase 1 dose-escalation trial. Lancet 2015;385:517-28.

36. Hu Y, Sun J, Wu Z, et al. Predominant cerebral cytokine release syndrome in CD19-directed chimeric antigen receptor-modified T cell therapy. J Hematol Oncol 2016;9:70.

37. Taraseviciute A, Tkachev V, Ponce R, et al. Chimeric Antigen Receptor T Cell-Mediated Neurotoxicity in Nonhuman Primates. Cancer Discov 2018;8:750-63.

38. Gust J, Hay KA, Hanafi LA, et al. Endothelial Activation and Blood-Brain Barrier Disruption in Neurotoxicity after Adoptive Immunotherapy with CD19 CAR-T Cells. Cancer Discov 2017;7:1404-19.

39. Bonifant CL, Jackson HJ, Brentjens RJ, et al. Toxicity and management in CAR T-cell therapy. Mol Ther Oncolytics 2016;3:16011.

40. Teachey DT, Bishop MR, Maloney DG, et al. Toxicity management after chimeric antigen receptor $\mathrm{T}$ cell therapy: one size does not fit 'ALL'. Nat Rev Clin Oncol 2018;15:218.

41. Fan F, Zhao W, Liu J, et al. Durable remissions with BCMA-specific chimeric antigen receptor (CAR)modified T cells in patients with refractory/relapsed multiple myeloma. J Clin Oncol 2017;35. doi: 10.1200/ JCO.2017.35.18_suppl.LBA3001

42. Brudno JN, Maric I, Hartman SD, et al. T Cells Genetically Modified to Express an Anti-B-Cell Maturation Antigen Chimeric Antigen Receptor Cause Remissions of Poor-Prognosis Relapsed Multiple Myeloma. J Clin Oncol 2018;36:2267-80.

43. Ali SA, Shi V, Maric I, et al. T cells expressing an anti-Bcell maturation antigen chimeric antigen receptor cause remissions of multiple myeloma. Blood 2016;128:1688-700.

44. Tasian SK. Acute myeloid leukemia chimeric antigen receptor T-cell immunotherapy: how far up the road have we traveled? Ther Adv Hematol 2018;9:135-48.

45. Castellarin M, Watanabe K, June CH, et al. Driving cars 
to the clinic for solid tumors. Gene Ther 2018;25:165-75.

46. Brown CE, Alizadeh D, Starr R, et al. Regression of Glioblastoma after Chimeric Antigen Receptor T-Cell Therapy. N Engl J Med 2016;375:2561-9.

47. Nellan A, Rota C, Majzner R, et al. Durable regression of Medulloblastoma after regional and intravenous delivery of anti-HER2 chimeric antigen receptor T cells. J Immunother Cancer 2018;6:30.

48. Priceman SJ, Tilakawardane D, Jeang B, et al. Regional Delivery of Chimeric Antigen Receptor-Engineered T Cells Effectively Targets HER2. Clin Cancer Res 2018;24:95-105.

49. Slaney CY, von Scheidt B, Davenport AJ, et al. Dualspecific Chimeric Antigen Receptor T Cells and an Indirect Vaccine Eradicate a Variety of Large Solid Tumors in an Immunocompetent, Self-antigen Setting. Clin Cancer Res 2017;23:2478-90.

doi: $10.21037 /$ sci.2018.11.05

Cite this article as: Wang $\mathrm{J}, \mathrm{Hu} \mathrm{Y}$, Huang $\mathrm{H}$. Current development of chimeric antigen receptor T-cell therapy. Stem Cell Investig 2018;5:44.
50. $\mathrm{Hu} \mathrm{Y}, \mathrm{Wu} \mathrm{Z}$, Luo Y, et al. Potent Anti-leukemia Activities of Chimeric Antigen Receptor-Modified T Cells against CD19 in Chinese Patients with Relapsed/Refractory Acute Lymphocytic Leukemia. Clin Cancer Res 2017;23:3297306.

51. John LB, Devaud C, Duong CP, et al. Anti-PD-1 antibody therapy potently enhances the eradication of established tumors by gene-modified T cells. Clin Cancer Res 2013;19:5636-46.

52. Tanoue K, Rosewell Shaw A, Watanabe N, et al. Armed Oncolytic Adenovirus-Expressing PD-L1 Mini-Body Enhances Antitumor Effects of Chimeric Antigen Receptor T Cells in Solid Tumors. Cancer Res 2017;77:2040-51.

53. Rosewell Shaw A, Porter CE, Watanabe N, et al. Adenovirotherapy Delivering Cytokine and Checkpoint Inhibitor Augments CAR T Cells against Metastatic Head and Neck Cancer. Mol Ther 2017;25:2440-51. 